\title{
FLOW-INDUCED INSTABILITY OF MULTI-LAYERED ANISOTROPIC PIPELINES
}

\author{
D.G. PAVLOU \\ Department of Mechanical and Structural Engineering and Materials Science, University of Stavanger, Norway.
}

ABSTRACT

A numerical formulation of flow-induced instability modelling of laminated anisotropic pipelines is derived. The analysis is based on fluid-structure interaction equations and FEA. Taking into account the flow parameters and the material properties, critical flow velocities causing instability are calculated for fibre-reinforced polymeric (FRP) pipelines resting on elastic supports. A parametric study of the effect of fibre orientation, stiffness of elastic supports and span length between supports is carried out. The results are commented and discussed.

Keywords:critical velocity, FEA, flow induced instability, laminated pipelines.

\section{INTRODUCTION}

In the last decade, there is a rapid increase of the use of fibre-reinforced polymeric materials (FRP) in pipeline installations. Their superior behaviour (Fig. 1) in corroded environment and fatigue loading as well as their high strength and low density, decrease significantly the maintenance cost of pipelines made from FRP materials [1-3]. Apart from the benefits on maintenance issues, the high stiffness of the laminated pipe wall yields superior mechanical behaviour in flow-induced instability conditions. Since the fluid-flow causes compressive axial forces [4-6], high values of the bending stiffness increase the critical values of flow velocity and improve the structural integrity of the pipeline. Until today, most of the existing theoretical tools for flow-induced instability analysis have been developed for steel pipelines [7]. However, the above methodologies cannot be applied to FRP pipelines due to the anisotropy of their material. In the present work, finite element (FE) analysis is carried out for critical flow velocity estimation of filament wound, multi-layered FRP pipelines resting on elastic supports.

\section{MOTION EQUATION OF MULTI-LAYERED FILAMENT WOUND FRP PIPES}

Taking into account the interaction of the pipe wall with the fluid (Fig. 2), the motion equation of filament wound FRP pipe is given by:

$$
S_{e q} \frac{\partial^{4} w(x, t)}{\partial x^{4}}+M U^{2} \frac{\partial^{2} w(x, t)}{\partial x^{2}}+2 M U \frac{\partial^{2} w(x, t)}{\partial x \partial t}+(M+m) \frac{\partial^{2} w(x, t)}{\partial t^{2}}=0
$$

where $M, m$ are the mass per unit length for the liquid and the pipe's material respectively, $U$ is the mean velocity of the liquid, $w(x, t)$ is the elastic deflection of the pipe, and $S_{e q}$ is the equivalent bending stiffness of the pipe for bending moment calculation, i.e.

$$
M(x)=S_{e q} \frac{d^{2} w}{d x^{2}}
$$




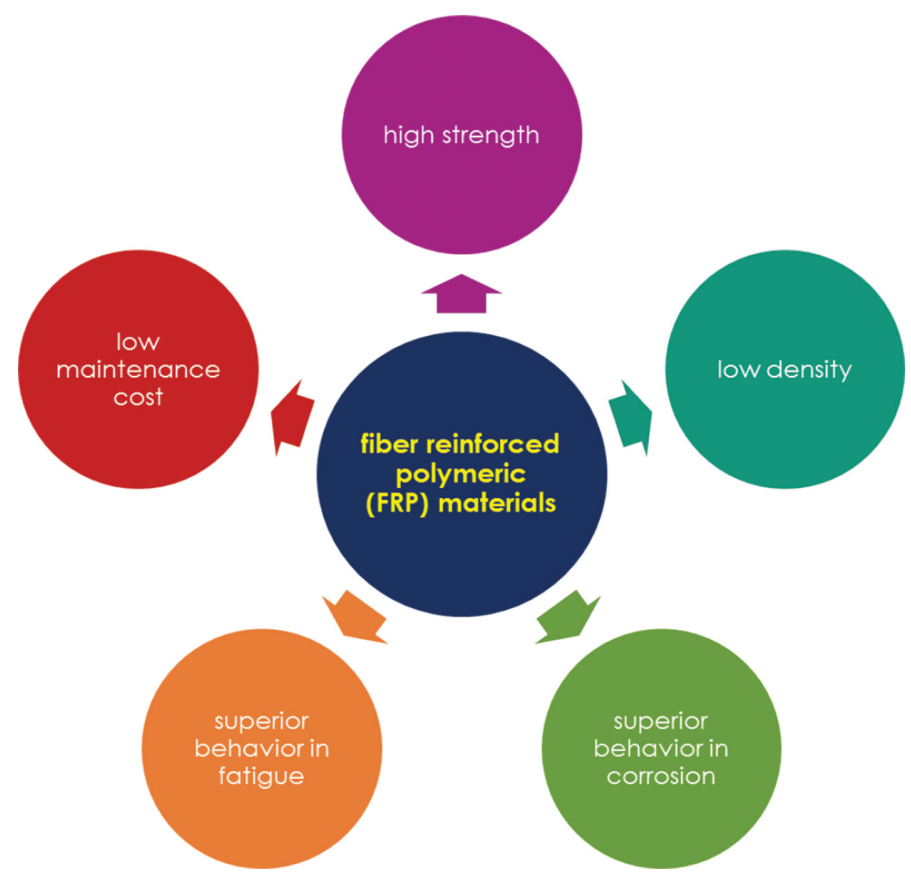

Figure 1: Advantages of the use of FRP materials in pipeline installations.
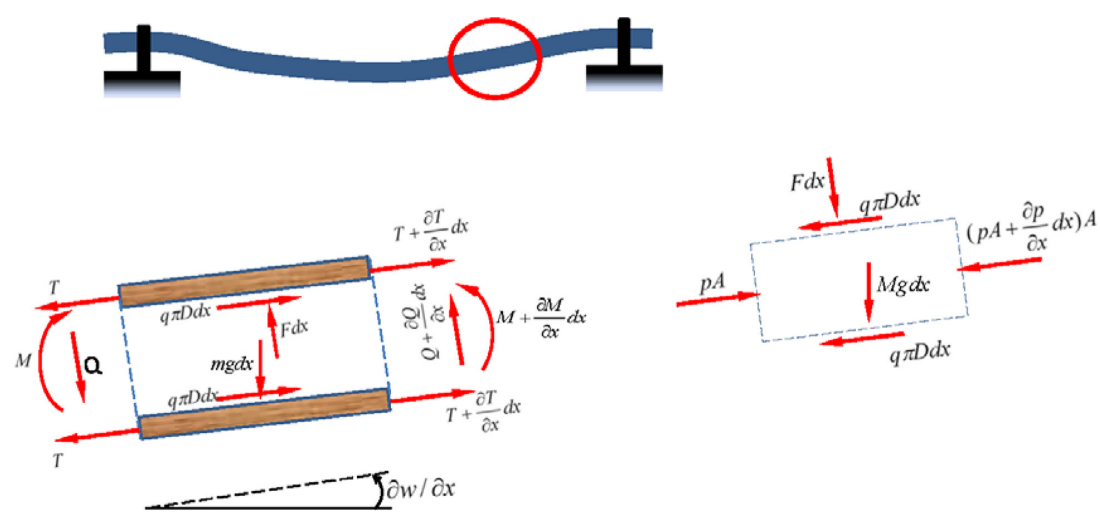

Figure 2: Interaction of the pipe wall with the fluid.

given by $[8,9]$

$$
S_{e q}=\frac{\pi D}{2}\left(\frac{D}{2 a_{11}}+\frac{1}{d_{11}}\right)
$$

In the above equations $M(x)$ is the bending moment, and $D$ is the diameter of the pipe. The parameters $\alpha_{11}, d_{11}$ can be calculated by the inversion of the stiffness matrix [ABD] of the 
laminate $[8,9]$. Their values depend on the mechanical properties E1, E2, G12, v12 in the principal directions, as well as on the fibre orientation angle $\vartheta$. Trying [10] into the above equation the solution

$$
w(x, t)=\operatorname{Re}\left[u(x) e^{i \omega t}\right]
$$

the following formula can be obtained:

$$
S_{e q} \frac{d^{4} u(x)}{d x^{4}}+M U^{2} \frac{d^{2} u(x)}{d x^{2}}+2 M U \omega i \frac{d u(x)}{d x}-(M+m) \omega^{2} u(x)=0
$$

\section{ELEMENT EQUATION OF A PIPE SEGMENT}

Following a standard mathematical procedure the differential eqn (5) can be transformed to the following matrix differential equation of the first order:

$$
\frac{d}{d x}\{Y\}=[\Omega] \cdot\{Y\}
$$

where

$$
\{Y\}=\left\{u(x) \quad u^{\prime}(x) \quad u^{\prime \prime}(x) \quad u^{\prime \prime \prime}(x)\right\}^{T}
$$

and

$$
[\Omega]=\left[\begin{array}{cccc}
0 & 1 & 0 & 0 \\
0 & 0 & 1 & 0 \\
0 & 0 & 0 & 1 \\
\frac{(M+m) \omega^{2}}{S_{e q}} & \frac{-2 M U \omega i}{S_{e q}} & \frac{-M U^{2}}{S_{e q}} & 0
\end{array}\right]
$$

Therefore, the solution of the matrix eqn (6) can be written:

$$
\left\{Y_{2}\right\}=e^{[\Omega] L} \cdot\left\{Y_{1}\right\}
$$

where $L$ is the length of the pipe segment 1-2 and

$$
\begin{gathered}
\left\{Y_{2}\right\}=\left\{u(L) \quad u^{\prime}(L) \quad u^{\prime \prime}(L) \quad u^{\prime \prime \prime}(L)\right\}^{T} \\
\left\{Y_{1}\right\}=\left\{u(0) \quad u^{\prime}(0) \quad u^{\prime \prime}(0) \quad u^{\prime \prime \prime}(0)\right\}^{T}
\end{gathered}
$$

Taking into account the following relations of solid mechanics

$$
\begin{gathered}
\vartheta(x)=u^{\prime}(x) \\
\mathrm{m}(x)=S_{e q} \mathrm{u}^{\prime \prime}(x) \\
\mathrm{f}(x)=S_{e q} \mathrm{u}^{\prime \prime \prime}(x)
\end{gathered}
$$

where $\vartheta(x), m(x), f(x)$ is the slope, bending moment and shear force distribution, the matrix eqn (9) can now be written in the following form: 


$$
\left\{\begin{array}{c}
u_{2} \\
\vartheta_{2} \\
m_{2} / S_{e q} \\
f_{2} / S_{e q}
\end{array}\right\}=e^{[\Omega] L}\left\{\begin{array}{c}
u_{1} \\
\vartheta_{1} \\
m_{1} / S_{e q} \\
f_{1} / S_{e q}
\end{array}\right\}
$$

Let's assume that the matrix exponential $e^{[\Omega] L}$ is expressed by the following matrix

$$
e^{[\Omega] L}=\left[\begin{array}{llll}
c_{11} & c_{12} & c_{13} & c_{14} \\
c_{21} & c_{22} & c_{23} & c_{24} \\
c_{31} & c_{32} & c_{33} & c_{34} \\
c_{41} & c_{42} & c_{43} & c_{44}
\end{array}\right]
$$

Performing some rearrangements in eqn (15), the following expression can be obtained:

$$
[F]\left\{\begin{array}{l}
f_{1} \\
m_{1} \\
f_{2} \\
m_{2}
\end{array}\right\}=[U]\left\{\begin{array}{l}
u_{1} \\
\vartheta_{1} \\
u_{2} \\
\vartheta_{2}
\end{array}\right\}
$$

where

$$
\begin{aligned}
& {[F]=\left[\begin{array}{lllc}
c_{14} / S_{e q} & c_{13} / S_{e q} & 0 & 0 \\
c_{24} / S_{e q} & c_{23} / S_{e q} & 0 & 0 \\
c_{34} / S_{e q} & c_{33} / S_{e q} & 0 & -1 \\
c_{44} / S_{e q} & c_{43} / S_{e q} & -1 & 0
\end{array}\right]} \\
& {[U]=\left[\begin{array}{cccc}
-c_{11} & -c_{12} & -1 & 0 \\
-c_{21} & -c_{21} & 0 & -1 \\
-S_{e q} c_{31} & -S_{e q} c_{32} & 0 & 0 \\
-S_{e q} c_{41} & -S_{e q} c_{42} & 0 & 0
\end{array}\right]}
\end{aligned}
$$

Therefore, the following equation for a segment $1-2$ can be derived:

$$
\left\{\begin{array}{c}
f_{1} \\
m_{1} \\
f_{2} \\
m_{2}
\end{array}\right\}=[k]\left\{\begin{array}{l}
u_{1} \\
\vartheta_{1} \\
u_{2} \\
\vartheta_{2}
\end{array}\right\}
$$

where

$$
[k]=[F]^{-1}[U]
$$

\section{PIPELINE RESTING ON ELASTIC SUPPORTS}

Let's assume a pipeline resting on equispaced elastic supports (Fig. 3). The elastic supports can be linear axial springs with elastic constant ka and linear torsional springs with elastic constants kt. Since the length and the material properties of all pipe segments are equal, the 


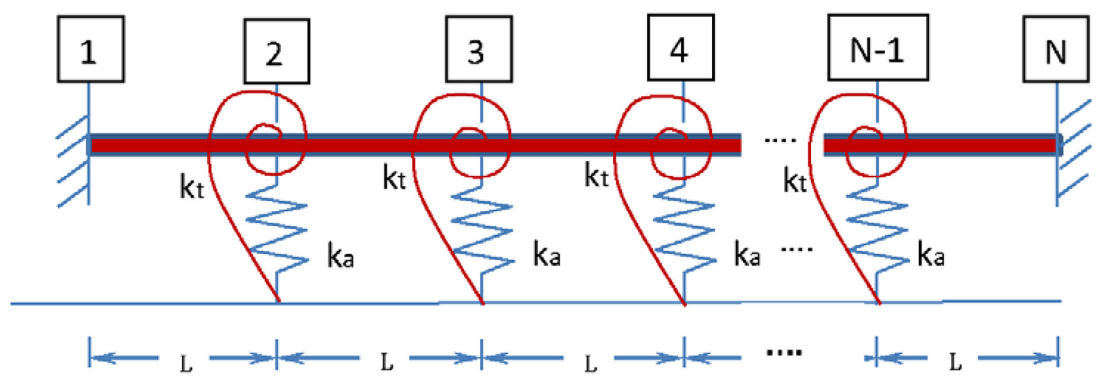

Figure 3: Nodes of a periodically multi-supported pipeline resting on elastic supports.

eqn (20) represents the element equation of each pipe element. Let's express the eqn (20) for two successive nodes $i, j$ in the following form:

$$
\left\{\begin{array}{c}
f_{i} \\
m_{i} \\
f_{j} \\
m_{j}
\end{array}\right\}=\left[\begin{array}{llll}
k_{11} & k_{12} & k_{13} & k_{14} \\
k_{21} & k_{22} & k_{23} & k_{24} \\
k_{31} & k_{32} & k_{33} & k_{34} \\
k_{41} & k_{42} & k_{43} & k_{44}
\end{array}\right]\left\{\begin{array}{c}
u_{i} \\
\vartheta_{i} \\
u_{j} \\
\vartheta_{j}
\end{array}\right\}
$$

Expansion of the above equation in global coordinates for any individual element $i$ - $j$ and superposition of the expanded element equations of all pipeline segments yield:

$\left\{\begin{array}{c}F_{1} \\ M_{1} \\ F_{2} \\ M_{2} \\ F_{3} \\ M_{3} \\ \vdots \\ F_{N-1} \\ M_{N-1} \\ F_{N} \\ M_{N}\end{array}\right\}=\left[\begin{array}{ccccccccccc}k_{11} & k_{12} & k_{13} & k_{14} & 0 & 0 & \ldots & 0 & 0 & 0 & 0 \\ k_{21} & k_{22} & k_{23} & k_{24} & 0 & 0 & \ldots & 0 & 0 & 0 & 0 \\ k_{31} & k_{32} & k_{33}+k_{11} & k_{34}+k_{12} & k_{13} & k_{14} & \ldots & 0 & 0 & 0 & 0 \\ k_{41} & k_{42} & k_{43}+k_{21} & k_{44}+k_{22} & k_{23} & k_{24} & \ldots & 0 & 0 & 0 & 0 \\ 0 & 0 & k_{31} & k_{32} & k_{33}+k_{11} & k_{34}+k_{12} & k_{13} & k_{14} & 0 & 0 & 0 \\ 0 & 0 & k_{41} & k_{42} & k_{43}+k_{21} & k_{44}+k_{22} & k_{23} & k_{24} & 0 & 0 & 0 \\ \vdots & \vdots & \vdots & \vdots & \vdots & \vdots & \vdots & \vdots & \vdots & \vdots & \vdots \\ 0 & 0 & 0 & 0 & 0 & k_{31} & k_{32} & k_{33}+k_{11} & k_{34}+k_{12} & k_{13} & k_{14} \\ 0 & 0 & 0 & 0 & 0 & k_{41} & k_{42} & k_{43}+k_{21} & k_{44}+k_{22} & k_{23} & k_{24} \\ 0 & 0 & 0 & 0 & 0 & 0 & \ldots & k_{31} & k_{32} & k_{33} & k_{34} \\ 0 & 0 & 0 & 0 & 0 & 0 & \ldots & k_{41} & k_{42} & k_{43} & k_{44}\end{array}\right]\left\{\begin{array}{c}u_{1} \\ \vartheta_{1} \\ u_{2} \\ \vartheta_{2} \\ u_{3} \\ \vartheta_{3} \\ \vdots \\ u_{N-1} \\ \vartheta_{N-1} \\ u_{N} \\ \vartheta_{N}\end{array}\right\}$

or in an abbreviated form:

$$
\left\{F_{2 N x 1}\right\}=\left[G_{2 N x 2 N}\right]\left\{d_{2 N x 1}\right\}
$$

The above equation can also be written as

$$
\left[\left[G_{2 N x 2 N}\right]\left[-I_{2 N x 2 N}\right]\right]\left\{\begin{array}{l}
\left\{d_{2 N x 1}\right\} \\
\left\{F_{2 N x 1}\right\}
\end{array}\right\}=\left[0_{2 N x 1}\right]
$$


Equation (25) is a $2 N x 2 N$ algebraic system with respect to the following $4 N$ unknowns

$$
\left.\begin{array}{l}
\left\{d_{2 N x 1}\right\}=\left\{\begin{array}{lllllllllll}
u_{1} & \vartheta_{1} & u_{2} & \vartheta_{2} & u_{3} & \vartheta_{3} & \cdots & u_{N-1} & \vartheta_{N-1} & u_{N} & \vartheta_{N}
\end{array}\right\}^{T} \\
\left\{F_{2 N x 1}\right\}=\left\{\begin{array}{lllllllllll}
F_{1} & M_{1} & F_{2} & M_{2} & F_{3} & M_{3} & \cdots & F_{N-1} & M_{N-1} & F_{N} & M_{N}
\end{array}\right\}^{T}
\end{array}\right\}
$$

In order the algebraic system provided by eqn (25) to be solvable, it should be completed by $2 \mathrm{~N}$ more equations. Let's consider the multi-supported pipeline demonstrated in the Fig. 3 . The boundary conditions for this example are

$$
\left.\begin{array}{l}
u_{1}=0 \\
\vartheta_{1}=0 \\
F_{2}=k_{a} u_{2} \\
M_{2}=k_{t} \theta_{2} \\
F_{3}=k_{a} u_{3} \\
M_{3}=k_{t} \theta_{3} \\
\vdots \\
F_{N-1}=k_{a} u_{N-1} \\
M_{N-1}=k_{t} \theta_{N-1} \\
u_{N}=0 \\
\vartheta_{N}=0
\end{array}\right\}
$$

The above boundary conditions can be written in the following matrix form:

$$
\left[\left[A_{2 N \times 2 N}\right]\left[B_{2 N x 2 N}\right]\right]\left\{\begin{array}{l}
\left\{d_{2 N x 1}\right\} \\
\left\{F_{2 N x 1}\right\}
\end{array}\right\}=\left\{0_{2 N \times 1}\right\}
$$

where

$$
\left[A_{2 N \times 2 N}\right]=\left[\begin{array}{ccccccccccc}
1 & 0 & 0 & 0 & 0 & 0 & \cdots & 0 & 0 & 0 & 0 \\
0 & 1 & 0 & 0 & 0 & 0 & \cdots & 0 & 0 & 0 & 0 \\
0 & 0 & -k_{a} & 0 & 0 & 0 & \cdots & 0 & 0 & 0 & 0 \\
0 & 0 & 0 & -k_{t} & 0 & 0 & \cdots & 0 & 0 & 0 & 0 \\
0 & 0 & 0 & 0 & -k_{a} & 0 & \cdots & 0 & 0 & 0 & 0 \\
0 & 0 & 0 & 0 & 0 & -k_{t} & \cdots & 0 & 0 & 0 & 0 \\
\vdots & \vdots & \vdots & \vdots & \vdots & \vdots & \cdots & \vdots & \vdots & \vdots & \vdots \\
0 & 0 & 0 & 0 & 0 & 0 & \cdots & -k_{a} & 0 & 0 & 0 \\
0 & 0 & 0 & 0 & 0 & 0 & \cdots & 0 & -k_{t} & 0 & 0 \\
0 & 0 & 0 & 0 & 0 & 0 & \cdots & 0 & 0 & 1 & 0 \\
0 & 0 & 0 & 0 & 0 & 0 & \cdots & 0 & 0 & 0 & 1
\end{array}\right]
$$




$$
\left[B_{2 N \times 2 N}\right]=\left[\begin{array}{ccccccccccc}
0 & 0 & 0 & 0 & 0 & 0 & \cdots & 0 & 0 & 0 & 0 \\
0 & 0 & 0 & 0 & 0 & 0 & \cdots & 0 & 0 & 0 & 0 \\
0 & 0 & 1 & 0 & 0 & 0 & \cdots & 0 & 0 & 0 & 0 \\
0 & 0 & 0 & 1 & 0 & 0 & \cdots & 0 & 0 & 0 & 0 \\
0 & 0 & 0 & 0 & 1 & 0 & \cdots & 0 & 0 & 0 & 0 \\
0 & 0 & 0 & 0 & 0 & 1 & \cdots & 0 & 0 & 0 & 0 \\
\vdots & \vdots & \vdots & \vdots & \vdots & \vdots & \cdots & \vdots & \vdots & \vdots & \vdots \\
0 & 0 & 0 & 0 & 0 & 0 & \cdots & 1 & 0 & 0 & 0 \\
0 & 0 & 0 & 0 & 0 & 0 & \cdots & 0 & 1 & 0 & 0 \\
0 & 0 & 0 & 0 & 0 & 0 & \cdots & 0 & 0 & 0 & 0 \\
0 & 0 & 0 & 0 & 0 & 0 & \cdots & 0 & 0 & 0 & 0
\end{array}\right]
$$

The combination of eqns (25)and (28) yields the following $4 N x 4 N$ algebraic system:

$$
\left[\begin{array}{ll}
{\left[G_{2 N \times 2 N}\right]} & {\left[-I_{2 N \times 2 N}\right]} \\
{\left[A_{2 N \times 2 N}\right]} & {\left[B_{2 N x 2 N}\right]}
\end{array}\right]\left\{\begin{array}{l}
\left\{d_{2 N x 1}\right\} \\
\left\{F_{2 N x 1}\right\}
\end{array}\right\}=\left\{0_{4 N x 4 N}\right\}
$$

The condition for non-trivial solution of the eqn (31) is:

$$
\operatorname{det}\left[\begin{array}{ll}
{\left[G_{2 N x 2 N}\right]} & {\left[-I_{2 N x 2 N}\right]} \\
{\left[A_{2 N x 2 N}\right]} & {\left[B_{2 N x 2 N}\right]}
\end{array}\right]=0
$$

The above equation can be solved numerically, providing the values of $\omega$ versus the values of $U$. Critical are the values of $U$ yielding the transition of $\operatorname{Im}\{\omega\}$ from a positive value (stability) to a negative one (instability).

\section{IMPLEMENTATION IN A REPRESENTATIVE EXAMPLE}

An S-Glass/Epoxy pipeline is resting on three linear equispaced springs with values $\mathrm{ka}=50 \mathrm{Kn} / \mathrm{m}$ and $\mathrm{kt}=114 \mathrm{KNm} / \mathrm{rad}$, respectively (Fig. 4). The interior diameter of the pipeline is $D=0.10 \mathrm{~m}$ and the laminated wall is composed of $\mathrm{N}=50$ layers with thickness $0.15 \mathrm{~mm}$. The material properties in the principal directions of each layer are $E_{1}=39 \mathrm{GPa}$, $E_{2}=8.6 \mathrm{GPa}, G_{12}=3.8 \mathrm{GPa}, v_{12}=0.2$, and the masses per unit length $\mathrm{m}$ and $\mathrm{M}$ have values

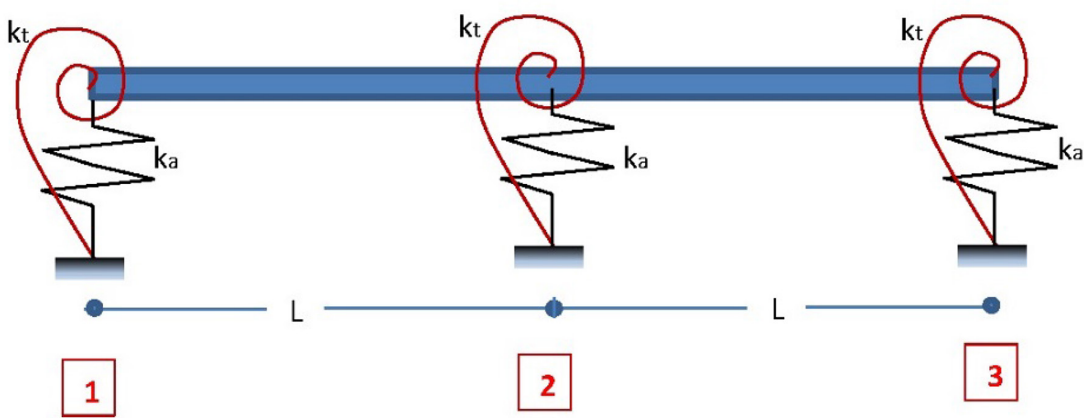

Figure 4: S-Glass/epoxy filament wound pipeline resting on a linear spring. 
$m=4.94 \mathrm{Kg} / \mathrm{m}$ and $M=7.85 \mathrm{Kg} / \mathrm{m}$. With the aid of the procedure described in the Fig. 5 the effect on the value of the critical flow velocity of the span between supports, fibre orientation and value of springs' elastic constant is going to be estimated. Taking into account the material data, the [abd] matrix [8,9] can be initially obtained for $\vartheta= \pm 30,45,60 \mathrm{deg}$. Then, the parameter $S_{e q}$ can be calculated by the eqn (3). The vectors $\{d\}$ and $\{F\}$ contain the following nodal displacements and forces corresponding to the example of Fig. 4:

$$
\{d\}=\left\{\begin{array}{llllll}
u_{1} & \vartheta_{1} & u_{2} & \vartheta_{2} & u_{3} & \vartheta_{3}
\end{array}\right\}
$$

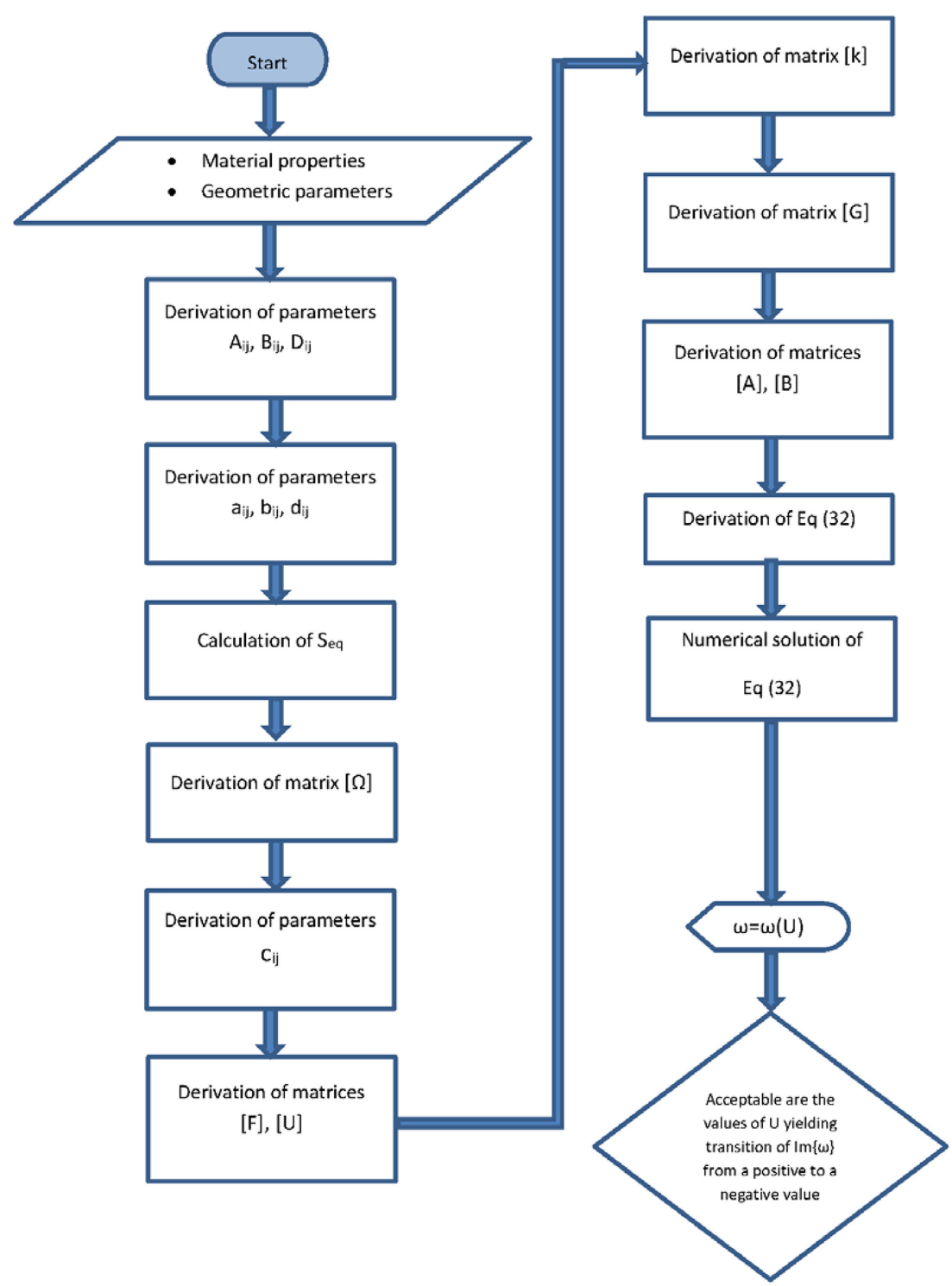

Figure 5: Procedure for derivation of critical flow velocity. 

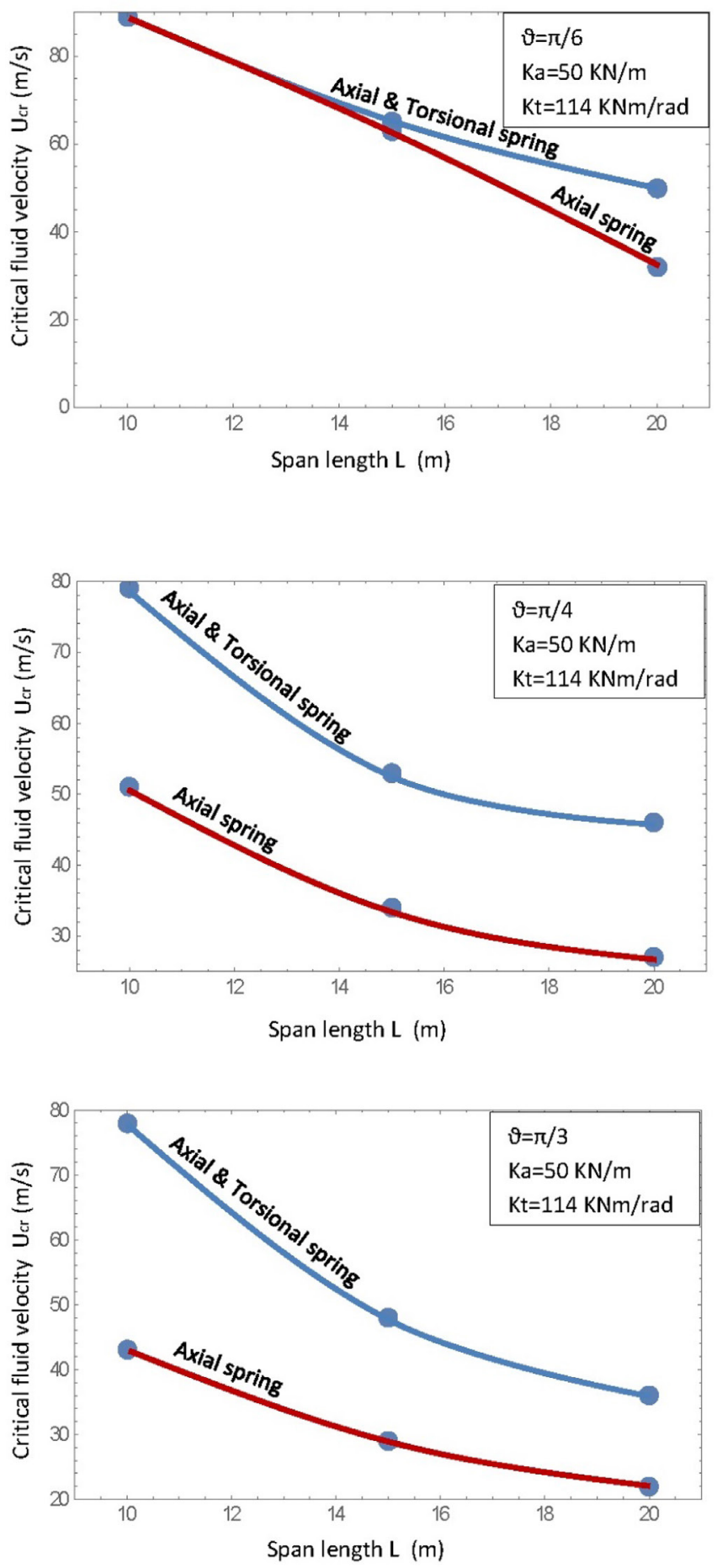

Figure 6: Critical flow velocity versus span length for a pipeline supported on axial or both axial and torsional springs: (a) Fibre orientation angle $\vartheta= \pm \pi / 6$, (b) Fibre orientation angle $\vartheta= \pm \pi / 4$, (c) Fibre orientation angle $\vartheta= \pm \pi / 3$. 


$$
\{F\}=\left\{\begin{array}{llllll}
F_{1} & M_{1} & F_{2} & M_{2} & F_{3} & M_{3}
\end{array}\right\}
$$

The boundary conditions are incorporated in the matrices $[\mathrm{A}]$ and $[\mathrm{B}]$ :

$$
\begin{gathered}
{[A]=\left[\begin{array}{cccccc}
-k_{a} & 0 & 0 & 0 & 0 & 0 \\
0 & -k_{t} & 0 & 0 & 0 & 0 \\
0 & 0 & -k_{a} & 0 & 0 & 0 \\
0 & 0 & 0 & -k_{t} & 0 & 0 \\
0 & 0 & 0 & 0 & -k_{a} & 0 \\
0 & 0 & 0 & 0 & 0 & -k_{t}
\end{array}\right]} \\
{[B]=\left[\begin{array}{cccccc}
1 & 0 & 0 & 0 & 0 & 0 \\
0 & 1 & 0 & 0 & 0 & 0 \\
0 & 0 & 1 & 0 & 0 & 0 \\
0 & 0 & 0 & 1 & 0 & 0 \\
0 & 0 & 0 & 0 & 1 & 0 \\
0 & 0 & 0 & 0 & 0 & 1
\end{array}\right]}
\end{gathered}
$$

The matrix $[G]$ can be obtained by the implementation of eqn (23) to the considered example. Therefore, its members contain functions of the variables $U$ and $\omega$. Using the matrices $[A],[B],[G]$, the eqn (32) can be derived yielding a correlation of $U$ versus $\omega$. For three values of fibre orientation angle $\vartheta= \pm \pi / 6, \pi / 4, \pi / 3 \mathrm{rad}$, the eqn (32) is solved numerically for a pipeline containing: (a) only axial springs, and (b) axial and torsional springs. For both cases, the solution is performed for three values of span length $\mathrm{L}=10,15,20 \mathrm{~m}$. Starting from a small initial value $U=1.0 \mathrm{~m} / \mathrm{s}$ for the flow velocity which is changed incrementally with step $1.0 \mathrm{~m} / \mathrm{s}$, the eigen-frequency $\omega$ is calculated using the standard commercial program Mathematica ${ }^{\circledR}$. The critical flow velocity values corresponding to the transition of $\operatorname{Im}\{\omega\}$ from a positive value (stability) to a negative one (instability) are demonstrated in the Fig. $6 \mathrm{a}-\mathrm{c}$. It should be noted that the numerical results indicated many changes of $\operatorname{Im}\{\omega\}$ from positive to negative values. Therefore, the pipeline has more than one critical flow velocities. In the Fig.5a-c, only the first critical flow velocity is adopted.

\section{CONCLUSIONS}

According to the above results the following main conclusions can be obtained:

(a) Short span length yields an increase of the critical flow speed, thus improving the dynamic stability of the pipeline.

(b) For the smallest fibre orientation angle $\vartheta= \pm \pi / 6$, the critical flow velocity increases smoothly for small values of span length. For higher values of the fibre orientation angle $\vartheta= \pm \pi / 4, \pm \pi / 3$ the increase of the critical flow velocity for small span length is rapid. This behaviour can be justified by the fact that small values of fibre orientation angle increase significantly the bending stiffness of the pipeline, and therefore dominate its dynamic stability.

(c) The superposition of both axial and torsional springs shifts upwards the curve of the critical fluid velocity vs span length. Since small fibre orientation angles dominate the 
bending stiffness, the effect of axial and torsional springs is small for $\vartheta= \pm \pi / 6$ and considerable for $\vartheta= \pm \pi / 4$ and $\pm \pi / 3$. For the last fibre orientation values, the value of critical flow velocity tends to be saturated for long span length.

(d) For large values of span length there is a divergence between the curves of Axial and Axial-Torsional springs for $\vartheta= \pm \pi / 6$, while for $\vartheta= \pm \pi / 3$ there is a convergence. Therefore, for flexible pipelines $(\vartheta= \pm \pi / 6$ ) the dynamic stability is dominated by the span length, while for stiff pipelines $(\vartheta= \pm \pi / 3)$ the dynamic stability is dominated by the fibre orientation angle.

\section{REFERENCES}

[1] Jin-Xing, S., Toshiaki, N., Xiao-Wen, L. \& Qing-Qing, N. Wave propagation in thefilament-wound composite pipes conveying fluid: Theoretical analysis for structural health monitoring applications.Composite Science and Technology, 98, pp. 9-14, 2014. http://dx.doi.org/10.1016/j.compscitech.2014.04.018

[2] Pavlou, D.G. Undamped vibration of laminated FRP pipes in water hammer conditions. ASME.Journal of Offshore Mechanics Arctic Engineering, 137(6), pp. 1-8, 2015. http://dx.doi.org/10.1115/1.4031669

[3] Ansari, R., Alisafaei, F. \& Ghaedi, P. Dynamic analysis of multi-layered filamentwoundcomposite pipes subjected to cyclic internal pressure and cyclic temperature. Composite Structures, 92, pp. 1100-1109, 2010. http://dx.doi.org/10.1016/j.compstruct.2009.09.058

[4] Kheiri, M. \& Païdoussis, M.P. On the use of generalized Hamilton's principle for the derivation of the equation of motion of a pipe conveying fluid. Journal of Fluids and Structures, 50, pp. 18-24, 2014. http://dx.doi.org/10.1016/j.jfluidstructs.2014.06.007

[5] Païdoussis, M.P. Fluid-Structure Interactions: Slender Structures and Axial Flow, AcademicPress: London, pp. 65-97, 2014.

[6] Kheiri, M., Païdoussis, M.P., Del Pozo, G.C. \& Amabili, M. Dynamics of a pipe conveying fluidflexibly restrained at the ends. Journal of Fluids and Structures, 49, pp. 360-385, 2014. http://dx.doi.org/10.1016/j.jfluidstructs.2013.11.023

[7] Nakamura, T., Kaneko, S., Inada, F., Kato, M., Ishihara, K., Nishihara, T., Mureithi, N.W. \& Langthjem, M.A. Flow-Induced Vibrations, Academic Press: London, pp. 157-170, 2014.

[8] Pavlou, D.G., Composite Materials in Piping Applications, Destech Publications: Lancaster, pp.105-168, 2013.

[9] Pavlou, D.G. \& Nergaard, A.I. Finite element analysis of FRP pipelines dynamic stability. InBoundary Elements and other Mesh Reduction Methods XXXVIII, eds. Cheng, A.H.D \& Brebbia, C.A, WIT Press: Southampton and Boston,WIT Transactions onModelling and Simulation, 61, pp. 139-151, 2015. http://dx.doi.org/10.2495/bem380111

[10] Zaitsev,V.F. \& Polyanin, A.D. Handbook of Exact Solutions for Ordinary Differential Equations,CRC Press: Florida, pp. 641-686, 2003. 